\title{
Fatigue behavior of 590MPa Tensile Strength galvanneal coated sheet steel laser welded blanks
}

\author{
Shrikant P. Bhat ${ }^{1, *}$, Ronald Soldaat ${ }^{1}$, and Gagan Tandon $^{2}$ \\ ${ }^{1}$ ArcelorMittal Global R\&D, 3001 East Columbus Drive, East Chicago, IN 46312, USA \\ ${ }^{2}$ ArcelorMittal Tailored Blanks, 8650 Mt Elliot, Detroit, MI 48211, USA
}

\begin{abstract}
The use of Laser Welded Blanks (LWBs) with different grade/gauge combinations in automotive body structures is well established; however, the acceptance of LWBs in fatigue critical chassis and underbody components has been slower because of lack of reliable models for durability assessment of laser welded joints (LWJ). Most prior fatigue studies of LWBs are carried out in tension - tension loading mode, making it difficult to relate it to the cyclic deformation and fatigue behaviors of the substrate steel grade. In contrast, in this study, LWJ is conceptualized as a "notch" to estimate the local stresses from the strain - life data of the parent grade and the fatigue notch concentration factor $\left(\mathrm{K}_{\mathrm{f}}\right)$ is estimated from the nominal stress values of LWJ. The method is illustrated with strain controlled fatigue data for $1.4 \mathrm{~mm}$ galvanneal coated $590 \mathrm{MPa}$ steel and fully reversed, fatigue data for homogeneous and heterogeneous LWB combinations. The results indicate that for both homogeneous and heterogeneous LWJ configurations, $\mathrm{K}_{\mathrm{f}}$ increases with fatigue life, but tends to saturate at life levels greater than about million cycles. Considering $10^{5}$ cycles to failure as an example, $K_{f}$ is estimated as 1.07 for the homogeneous and 1.25 for the heterogeneous combinations.
\end{abstract}

\section{Introduction}

\subsection{Industrial Relevance}

Over the last several decades, increasingly stringent safety requirements, demands for higher fuel economy and the need to reduce greenhouse gas emissions for cleaner environment all at a competitive price (raw material and conversion cost included) have spurred the development of new high strength steel grades and more importantly newer processes to better utilize steel grades in automotive applications. One such technology introduced in the early 1980's for better steel utilization is Tailor Welded Blanks (TWB). While there are many welding methods to produce TWBs, this report will focus on tailored blanks produced by laser welding (LWB). The advantages of laser welding to produce TWBs include ease of welding different steels (grade, thickness, and coating), narrow weld and heat affected zone (HAZ), relatively low geometric distortion, relatively high welding speed and high penetration [1]. Over the last several years, LWB has gained wide acceptance in body structure applications (for example, door rings, door inner panels, B-pillars, sills, floors, etc.). However, the use of LWBs in chassis, suspension and other underbody components (for example, subframes) has been rather minimal. The paucity stems from the fact that while thinner (or lower strength) material can be placed in areas with less critical stiffness, strength and crashworthiness requirements, it may be difficult to find "less critical" areas of a component designed for fatigue/durability resistance [2]. In addition, many of the underbody components tend to be heavier gauge hot rolled or hot-rolled \& coated products for which laser-welding (to produce LWB) may not be as straight forward.

This research was conducted to promote high strength steels in front or rear subframes of a passenger vehicle via LWB to be weight competitive with alternate materials. Road-load durability assessment is a critical performance evaluation step for subframes. Following the preliminary part design and manufacturing feasibility evaluation, it was necessary to address the durability concerns, both with respect to component testing and a method of estimating it via finite element analysis (FEA) codes for the LWB joint. This report summarizes the fatigue test results and an estimation of notch effects due to LWB in support of this overall effort on one steel grade of interest to the sub-frame.

\subsection{Technical Challenges - Conceptualizing LWB as a Notch}

Fatigue failures almost always initiate at geometric discontinuities (shape change, thickness change or joints) that act as stress or strain concentrations. In general, under cyclic loading, the geometric discontinuities are less effective than predicted by $\mathrm{K}_{\mathrm{t}}$ (static theoretical stress concentration factor) in raising the local stress or strain, and therefore, the effects of notches in fatigue is usually described by $\mathrm{K}_{\mathrm{f}}$, Fatigue Notch Factor or Fatigue Strength Reduction Factor. In this investigation, LWB zone is conceptualized as a "notch" and the focus is to determine $\mathrm{K}_{\mathrm{f}}$ for LWB. 
The fatigue notch factor $K_{f}$ is the ratio of un-notched fatigue strength to notched fatigue strength at a given life level [3]:

$$
\mathrm{K}_{\mathrm{f}}=\left(\boldsymbol{\sigma}_{\mathrm{UNNOTCHED}}\right) /\left(\boldsymbol{\sigma}_{\text {NOTCHED }}\right)
$$

Although this concept of $\mathrm{K}_{\mathrm{f}}$ is relatively simple, experimental determination is difficult because it depends on notch geometry (LWB zone), life level, loading mode, load ratio, etc making the applicability of data from one experimental protocol to another questionable and often difficult.

In the automotive industry with emphasis on finitelife design, the use of low cycle fatigue (LCF) concepts for component durability estimation is rather common. The focus is on crack initiation and the crack propagation life is ignored or considered to provide a safety factor against catastrophic failures. Therefore ideally, the estimation of $\mathrm{K}_{\mathrm{f}}$ should take into account the LCF behavior of the steel grade.

There have been many studies elucidating the effects of laser welding on fatigue behavior of steels; differences in laser welding technology applied to part assembly and that used in tailored blank production should be kept in mind in reviewing these results. For example, Wang and Ewing [4] showed that the tensile fatigue strengths of laser welds are superior to those of spot welds in lap shear joint configuration for DQSK steel. Daneshpour et al [5] showed that laser spot welds exhibited better fatigue life compared to spot welds, but only at "high load" levels (or short life) for dual phase 780 but not in DQSK type of steel. Asim et al [6] found that in laser welded lap shear joints of HSLA steel, crack initiation was near the boundary of the base metal and heat affected zone (HAZ) under static conditions, but initiated at pre-existing crack tips under cyclic loading (both low cycle and long-life regimes). Anand et al [2] reported that zinc-coated steels with differential thickness TWB joints exhibited a lower fatigue limit compared to similar TWB joints of uncoated materials. Most of these earlier studies were carried out in tensiontension fatigue mode (often with load ratio $\mathrm{R}=0.1$ ) and most often these studies do not report the LCF behavior of the parent grade of steel, making any generalization extremely difficult. In the current program, LCF behavior of the parent steel grade and an estimation of $\mathrm{K}_{\mathrm{f}}$ for two different LWB combinations will be emphasized.

\section{Experimental Details}

\subsection{Materials and Experimental Procedure}

The steel grade used in this study is GA 590R, indicating a minimum tensile strength of $590 \mathrm{MPa}$ with a relatively high yield ratio (Yield strength/Tensile strength) and galvannealed (GA) coating produced via hot dip process. The coating and the substrate metallurgy details of producing such products can be found elsewhere [7,8]. Two commercially produced coils at $1.4 \mathrm{~mm}$ and 1.0 $\mathrm{mm}$ thicknesses were secured for the present study. The chemistry and the coating details of the product are listed in Table 1 while the tensile properties (determined following the JIS protocols) are listed in Table 2. No measurable differences were found for the two thicknesses.

Table 1. Chemistry and coating characteristics of GA 590R

\begin{tabular}{|c|c|c|c|c|c|c|c|c|}
\hline \multicolumn{7}{|c|}{ SUBSTRATE CHEMISTRY, (wt \%) } & \multicolumn{2}{|c|}{\begin{tabular}{|c}
$\begin{array}{c}\text { GA COATING } \\
\text { (Top/Bottom) }\end{array}$ \\
\end{tabular}} \\
\hline C & Mn & $\mathrm{Si}$ & $\mathbf{P}$ & $\mathbf{s}$ & $\mathrm{Al}$ & $\mathbf{N}$ & $\mathrm{wt}, \mathrm{g} / \mathrm{m}^{2}$ & $\% \mathrm{Fe}$ \\
\hline 0.104 & 2 & 0.13 & 0.014 & 0.001 & 0.02 & 0.005 & $43 / 38$ & 9/9 \\
\hline
\end{tabular}

Table 2. Tensile properties of GA 590R

\begin{tabular}{|c|c|c|}
\hline \multicolumn{2}{|c|}{ TENSILE TEST RESULTS (JIS No. 5 Trans verse) } \\
\hline $\begin{array}{c}\text { YIELD } \\
\text { STRENGTH, MPa }\end{array}$ & TENSILE & TOTAL \\
\hline 494 & 656 & 25 \\
\hline
\end{tabular}

Rectangular LWBs were produced in the homogeneous $(1.4 / 1.4 \mathrm{~mm})$ and heterogenous $(1.4 / 1.0$ $\mathrm{mm}$ ) combinations using a commercial $\mathrm{CO}_{2}$ laser. A cross-section of one of the laser welded joints is shown in Figure 1 and demonstrates good laser weld quality.

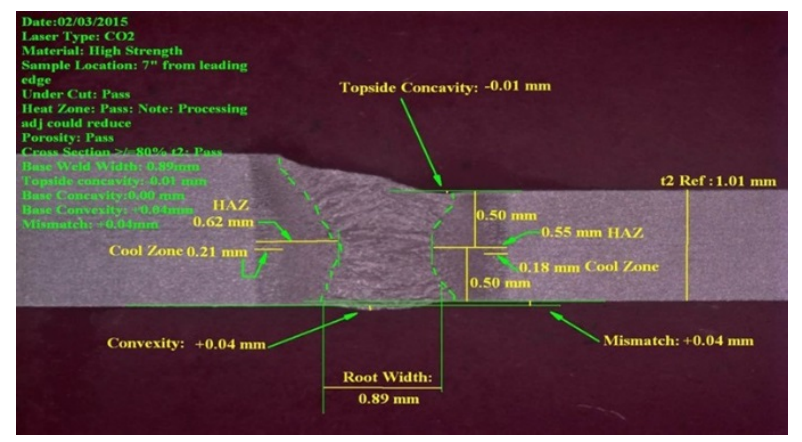

Fig. 1. Heterogeneous (1.4 / 1.0 mm) LWJ of GA 590R

The three primary areas of investigation included: a) Tensile and fatigue testing of monolithic GA 590R $(1.4 \mathrm{~mm})$; b) Tensile and fatigue testing of homogeneous LWB coupons (1.4/1.4 mm). and c) Tensile and fatigue testing of heterogenous LWB coupons. Tensile tests were carried out on an Instron machine and the axial, tension-compression, fully reversed $(\mathrm{R}=-1)$ fatigue tests were carried out on an MTS servo-hydraulic test system following the ASTM E 606 procedure adapted for thin sheet steels.

\section{Results and Discussion}

\subsection{Tensile test results}

Tensile test results are summarized in Table 3 for monolithic, as well as homogeneous, and heterogeneous LWB combinations (weld line perpendicular to loading direction). The data indicate that the laser welding process has very little influence on the yield and tensile strengths of the steel but it greatly reduces the "apparent" ductility parameters - total elongation (TE) and uniform elongation (UE) values. This is illustrated in Figure 2 via the engineering stress - strain curves for the three conditions investigated in this work. 
Table 3. Tensile test results for LWB of GA 590R

\begin{tabular}{|c|c|c|c|c|c|}
\hline DESCRIPTION & GAUGE, mm & YS, MPa & TS, MPa & TE, \% & UE, \% \\
\hline Monolithic & 1.4 & 494 & 656 & 24.7 & 12.9 \\
\hline Homogeneous & $1.4 / 1.4$ & 497 & 652 & 18.6 & 9.4 \\
\hline Heterogeneous & $1.4 / 1.0$ & 492 & 658 & 13.7 & 6.8 \\
\hline
\end{tabular}

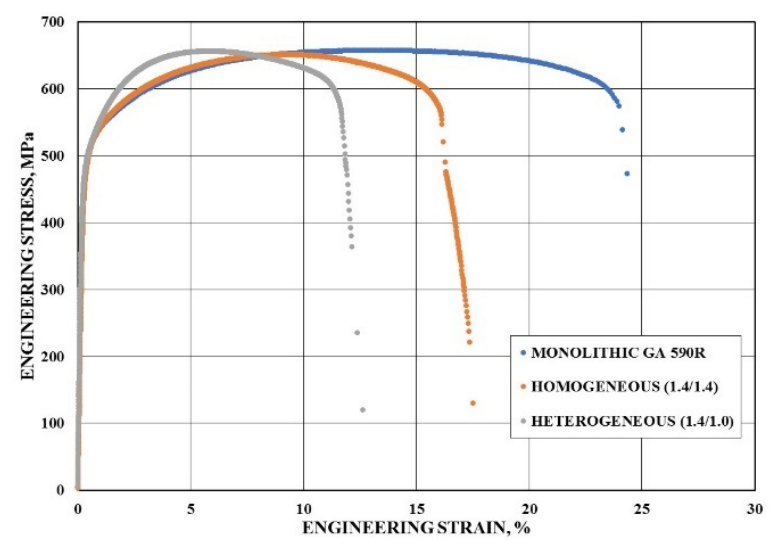

Fig.2. Engineering stress -strain curves for monolithic and two LWB GA 590R

The apparent loss of ductility is attributable to the higher hardness of the weld region in the center portion of the gauge length of the tensile coupon. The Vickers hardness profiles from the center of the weld for the three conditions are shown in Figure 3. The weld hardness $(380-400 \mathrm{VHN})$ is approximately twice that of the base metal $(190-210 \mathrm{VHN})$. The weld hardness values are reasonable with the fully martensitic structure with a steel chemistry of $0.1 \mathrm{C}$, and $2 \mathrm{Mn}$ (this hardness corresponds to a tensile strength of about $1220 \mathrm{MPa}$ ).

It should be noted that the weld and the HAZ in LWBs are comparatively narrow at about $2 \mathrm{~mm}$ (weld zone width about $1 \mathrm{~mm}$ and the HAZ width about $1 \mathrm{~mm}$, see Figure 1). This high hardness zone acts as a barrier to deformation during the tensile deformation. In other words, the martensite in the weld region is expected to be in the elastic regime, even when other portions of the specimen are well into the plastic deformation regime.

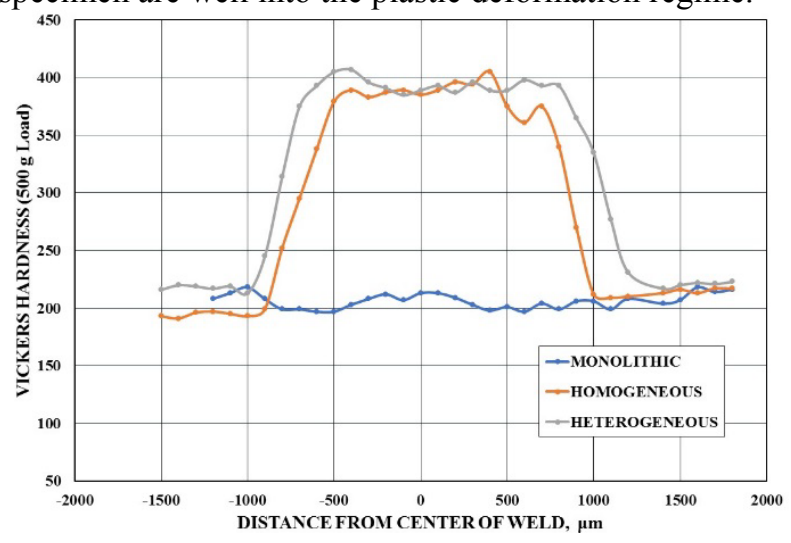

Fig 3. Microhardness profile of base steel and LWB coupons

Thus, the weld region may be considered to act as an artificial "grip" region, but the machine records the total extension and the original gauge length is used to calculate the engineering total elongation and uniform elongation values. Further, Figure 4 shows the fracture profiles of two LWB coupons after tensile testing with final failure occurring in the base metal, significantly far away from the weld zone, in both homogeneous and heterogeneous LWBs.

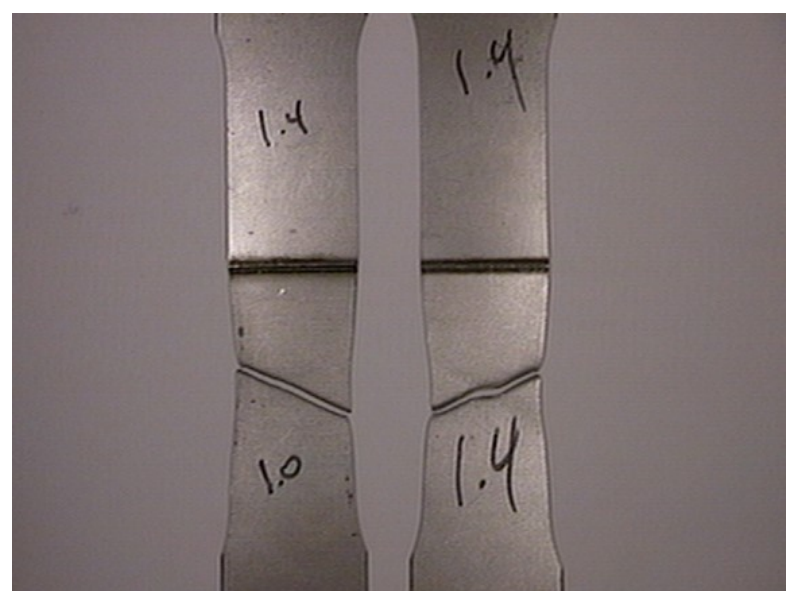

Fig 4. Fracture profile of Heterogeneous and homogeneous LWB tensile coupons

Therefore, it is emphasized that the ductility parameters determined using the normal tensile specimen protocols are "apparent" values and do not reflect the true values. The weld zone is narrow in LWB and the hardness remains unaffected in other areas. This "high" hardness of the weld zone concentrates the deformation zone away from the weld area and the final fracture occurs due to strain localization in the matrix. In this regard, it is important to highlight that in laser welding of GA 590R, the high hardness in the weld zone decreases gradually in the HAZ reaching that of the base metal without any observable softening (no valley in the hardness profile) in the HAZ. In contrast, many earlier research reports indicate that the tensile failures in LWBs with DP steels originate in the HAZ due to local softening or hardness valleys [9-11]. Such was not the case and no HAZ softening was noted in GA 590R after LWB production.

\subsection{Fatigue test results}

In this investigation, fatigue behavior of GA 590R is characterized under four different experimental protocols: a) Strain controlled fatigue characteristics of the base steel (monolithic); b) Load controlled fatigue behavior for base steel (monolithic); c) Load controlled fatigue behavior of homogeneous laser welded coupons (1.4 /1.4 mm combination) and d) Load controlled fatigue behavior of heterogeneous laser welded coupons (1.4/1.0 mm combination). These results are presented first and then the data are used to estimate the fatigue notch factors of LWB joints in this steel grade.

\subsubsection{Strain Controlled Low Cycle Fatigue (LCF) of GA $590 R$}

LCF characterization of the steel was carried out through axial, fully reversed, constant amplitude, total strain controlled tests; at least two specimens were tested at each strain level in the life range of $\sim 2000$ to $5,000,000$ cycles to failure. Both cyclic deformation and 
life aspects as determined through strain controlled tests are highlighted below.

Cyclic Response Curves: Macroscopic stability of a steel under fatigue loading can be represented as a plot of the stress needed to impose the strain limits as a function of the number of cycles elapsed throughout the life. Cyclic response curves (CRC) for GA 590R at three representative strain amplitudes are shown in Figure 5, in which the resistance to strain cycling is represented as the averages of the tensile and compressive maximum flow stress values (without regard to sign). Cycling continuously modifies the stress response throughout fatigue life at most strain amplitudes. At very low strain amplitude $(0.15 \%)$, the stress response is very stable through most of life. However, at higher strain amplitudes (for example, $0.6 \%$ ), specimens exhibit gradual softening throughout life and only a weak indication of saturation can be detected late in life (approximately at half-life).

Cyclic Stress - Strain (CSS) Curve: An appropriate stress - strain relationship is needed to account for the inelastic behavior of steel in a structure, for example near a notch. The results presented in Figure 5 indicate that cyclic loading can alter the stress - strain relationship significantly from its virgin form. This change in behavior is depicted in Figure 6 by comparing the monotonic and cyclic stress - strain behavior for low strain levels (up to $1 \%$ total strain). The CSS curve deviates from the monotonic curve significantly and shows a large cyclic softening in the yield point region, due to the removal of the yield point i.e. generation of fresh unpinned dislocations [12]. From an engineering perspective, the use of tensile stress - strain curve for fatigue analysis may lead to significant errors.

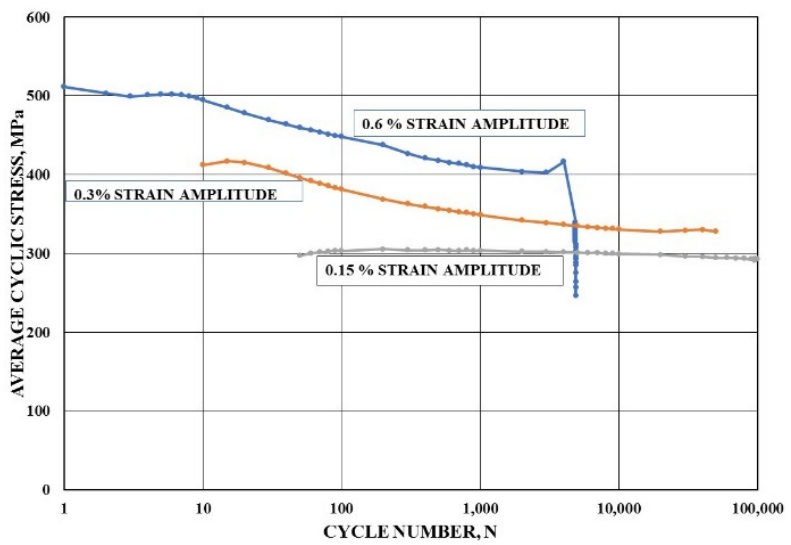

Fig. 5. Cyclic response curves at three representative strain amplitudes for GA 590R.

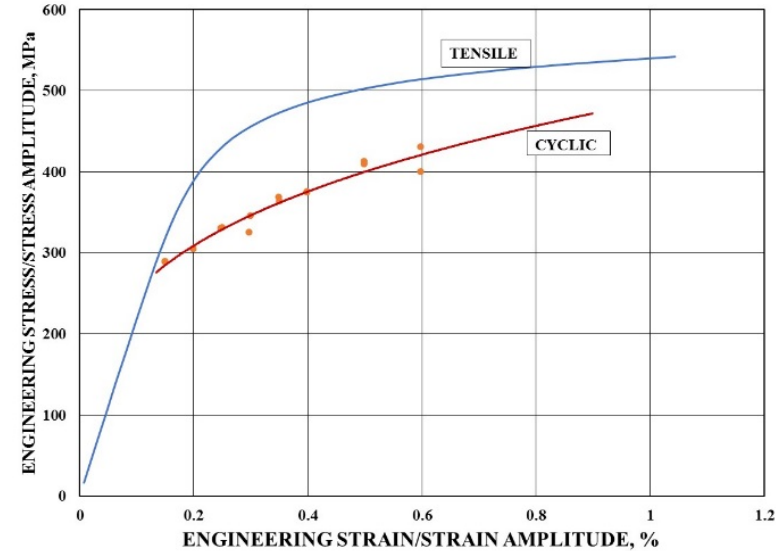

Fig. 6. Comparison of cyclic and tensile stress - strain curves for GA 590R

Strain - Life Curves: Strain - life data from constant amplitude tests for GA 590R are shown in Figure 7; this figure includes total strain amplitude (test control variable) as well as the component strain amplitudes (Plastic and Elastic) determined from approximately half-life hysteresis loops. The two component strains when plotted against fatigue life on a $\log -\log$ scale lead to straight lines and of course the total strain - life plot is not. Following the procedures detailed in ASTM E739, the data were analyzed with the fatigue life as the dependent variable and the plastic and elastic strain components as the independent variables to determine Fatigue Parameters, using the equations given below:

$$
\left(\Delta \varepsilon_{\mathrm{pl}} / 2\right)=\varepsilon_{\mathrm{f}}\left(2 \mathrm{~N}_{\mathrm{f}}\right)^{\mathrm{c}}
$$

Plastic Strain - Life (Coffin-Manson Equation)

$$
\left(\Delta \varepsilon_{\mathrm{el}} / 2\right)=\left(\sigma_{\mathrm{f}} / \mathrm{E}\right)\left(2 \mathrm{~N}_{\mathrm{f}}\right)^{\mathrm{b}}
$$

Elastic Strain - Life (Basquin Equation)

$$
(\Delta \sigma / 2)=\mathrm{k}^{\prime}\left(\Delta \varepsilon_{\mathrm{pl}} / 2\right)^{\mathrm{n}^{\prime}}
$$

Cyclic Stress - Strain Equation

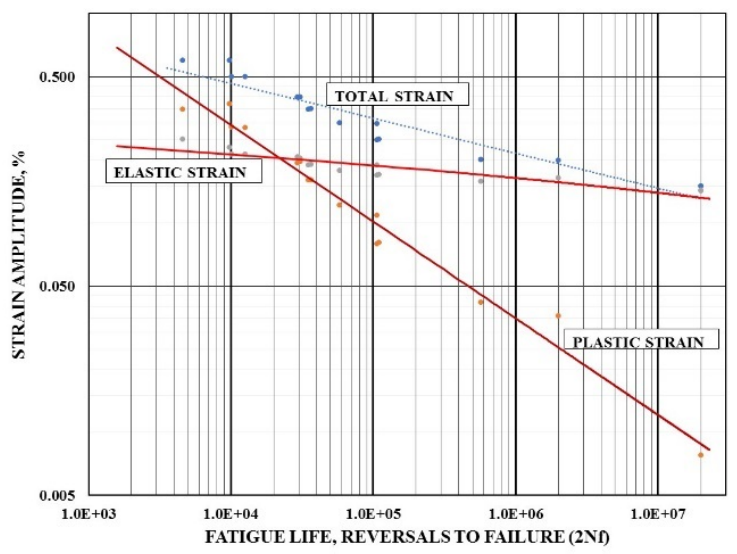

Fig. 7. Strain -Life fatigue plots for GA $590 \mathrm{R}$

The fatigue parameters determined from linear regression analyses of the data (on transformed logarithmic variables) are listed in Table 4. Correlation coefficients are also listed for each of the equations to indicate robustness of the data. It must be kept in mind that all the data come from one coil and one location and thus they do not reflect the variability within the grade. Comparison of data in Table 3 to those in Table 4 show that the cyclic yield strength of $376 \mathrm{MPa}$ is substantially 
lower than that of the tensile yield strength of $494 \mathrm{MPa}$ for this steel. Thus, the use of the tensile YS for calculations under fatigue loading situations may be too optimistic.

Table 4. Summary Strain - Life fatigue parameters for GA 590R

\begin{tabular}{|c|c|}
\hline 1. CYCLIC STRESS -STRAIN EQUATION & \\
\hline Cyclic Strength Coefficient, k', MPa & 681 \\
\hline Cyclic Strain Hardening Exponent, n' & 0.096 \\
\hline Correlation Coefficient, $\mathbf{R}^{2}$ & 0.85 \\
\hline Cyclic Yield Strength (CYS), MPa & 376 \\
\hline 2. PLASTIC STRAIN - LIFE EQUATION & \\
\hline Fatigue Ductility Coefficient, $\varepsilon_{f}^{\prime}$ & $\mathbf{0 . 2 8 2}$ \\
\hline Fatigue Ductility Exponent, c & -0.491 \\
\hline Correlation Coefficient, $\mathbf{R}^{2}$ & 0.98 \\
\hline 3. ELASTIC STRAIN - LIFE EQUATION & \\
\hline Fatigue Strength Coefficient, $\sigma_{f}^{\prime}, M P a$ & 849 \\
\hline Fatigue Strength Exponent, b & -0.069 \\
\hline Correlation Coeffcient, $\mathbf{R}^{2}$ & 0.86 \\
\hline
\end{tabular}

\subsubsection{High Cycle Fatigue (HCF) for monolithic GA $590 R$}

Long life fatigue data generated under stress control are shown in Figure 8 as the Stress - Life (S-N) plot with $10^{7}$ cycles taken as the run-outs (tests were terminated if the specimen survived this life level). The data refer to axial loading and fully reversed (Load Ratio, $\mathrm{R}=-1$ ) condition and the tests were run at a constant frequency of 20 or $30 \mathrm{~Hz}$. Using these data, fatigue limit is estimated to be $310 \mathrm{MPa}$ and a fatigue ratio (Fatigue Limit / Tensile Strength) of 0.47 .

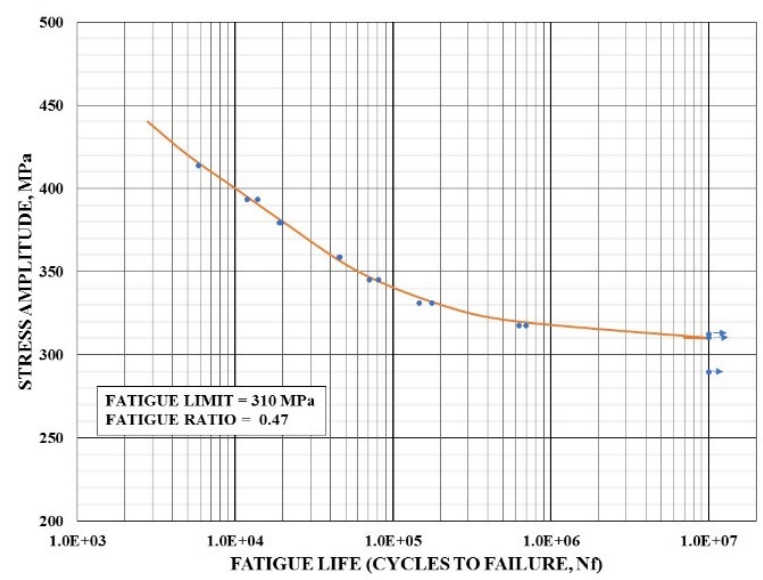

Fig. 8. S-N curve for monolithic GA 590R (1.4 mm)

\subsubsection{S-N Curve for Homogeneous LWB of GA 590R}

Attempts to carry out strain controlled fatigue tests under axial, fully reversed mode were not successful, because of excessive buckling due to stress concentration at the weld line. Therefore, only the load controlled fatigue tests were carried out for both homogeneous and heterogeneous laser welded coupons.
For all the fatigue tests, the loading axis was perpendicular to the weld line. Square cross section of the specimens and careful alignment (of load train) were critical to achieve fully reversed loading condition. A critical distinction of the current fatigue work on laser welded coupons is important to emphasize. Most prior fatigue work after laser welding [2, 4-6, 9-11] is carried out in tension - tension mode (typical load ratio, $\mathrm{R}=+$ $0.1)$ rather than in fully reversed condition $(R=-1)$ to minimize local buckling issues. However, it is well known that only the $\mathrm{R}=-1$ loading condition does not introduce significant mean stress at notch roots [13] during fatigue tests and makes it possible to relate these data to those of the smooth specimen (monolithic) strain -life data. Because of mean stress effects at the notch root, it is difficult to relate the data generated under other load ratios (for example $\mathrm{R}=0.1$ ) to smooth specimen LCF data (strain control tests are not usually carried out with mean strain). Thus, to the best of our knowledge, the current investigation is the first one to record the fatigue data for laser butt-welded joints under fully reversed, axial loading condition.

S-N data for homogeneous $(1.4 / 1.4 \mathrm{~mm})$ laser welded GA 590R coupons are presented in Figure 9. Estimated fatigue limit of $300 \mathrm{MPa}$ and the Fatigue Ratio of 0.46 are only slightly below that of the monolithic GA 590R ( $4 \%$ drop) and it may be concluded that the influence of laser butt welding on the axial, fully reversed fatigue is to decrease the fatigue limit by $10 \mathrm{MPa}$ or $\sim 4 \%$ for this steel.

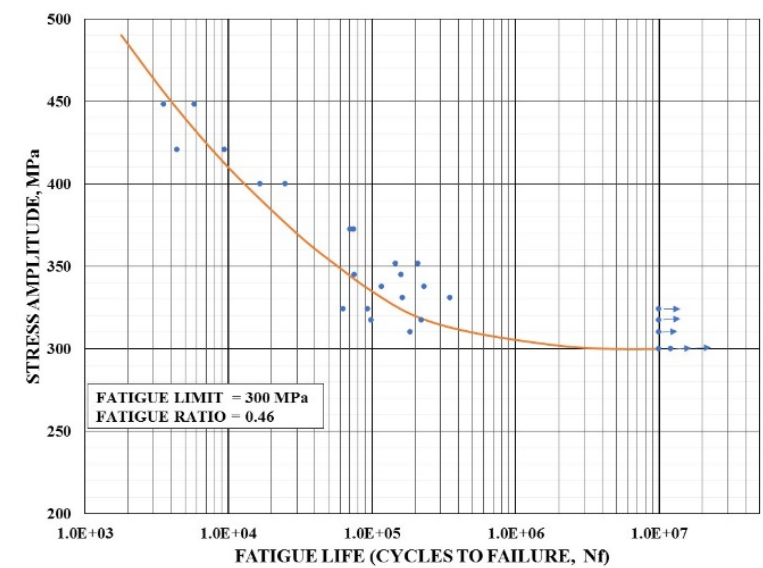

Fig.9. S-N curve for homogeneous LWB of GA 590R (1.4 / $1.4 \mathrm{~mm})$; Axial, fully reversed.

\subsubsection{S-N Curve for Heterogeneous Laser Welded GA $590 R$}

Stress controlled fatigue tests were repeated for heterogeneous $(1.4 \mathrm{~mm} / 1.0 \mathrm{~mm})$ laser welded GA 590R with the same test protocols. To maintain axiality and to avoid fractures in the specimen shoulders, it was necessary to add thin metal shims on the thinner side of the specimen. All the stress calculations are based on the thinner gauge section. The results are presented in Figure 10. Fatigue limit data for the three test conditions 
are summarized in Table 5. Estimated fatigue limit of $240 \mathrm{MPa}$ for the heterogeneous LWB and a fatigue ratio of 0.36 are substantially lower than those of the base material and the homogeneous LWB. This decrease can be attributed to the local stress concentration introduced by gauge differences at the weld. Keeping in mind that even for heterogeneous combination, tensile strengths are the same as that for the base material, this demonstrates the influence of local "notches" on the fatigue behavior of joints. This will be illustrated further in the following section.

Table 5. S-N data summary for GA590R

\begin{tabular}{|l|c|c|c|}
\hline \multicolumn{4}{|c|}{$\begin{array}{r}\text { FATIGUE LIMIT DATA FOR GA 590R LWB } \\
\text { (AXIAL, FULLY REVERSED, R = -1) }\end{array}$} \\
\hline SPECIMEN CONDITION & $\begin{array}{l}\text { FATIGUE LIMIT } \\
\left(10^{7} \text { CYCLES) }\right.\end{array}$ & TS, MPa & $\begin{array}{l}\text { FATIGUE RATIO } \\
\text { (Fatigue Limit /TS) }\end{array}$ \\
\hline MONOLITHIC & & & \\
\hline HOMOGENEOUS (1.4/1.4) & 310 & 656 & 0.47 \\
\hline HETEROGENEOUS (1.4/1.0) & 240 & 652 & 0.46 \\
\hline
\end{tabular}

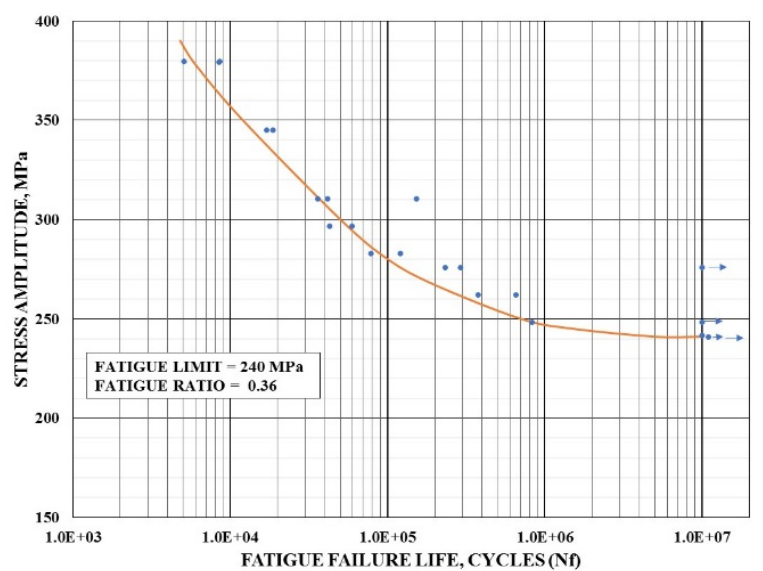

Fig 10. S-N curve for heterogeneous LWB $(1.4 / 1.0 \mathrm{~mm})$ of GA590R

\subsection{Estimation of Fatigue Notch Factor for LWB}

Ever since Wholer found almost 150 years ago that adding material to a railway axle might in fact make it weaker in fatigue, notch effects have been a thorny problem both for researchers and design engineers [14, 15]. The primary focus in notch fatigue research has been to estimate the local (notch root) stress $(\sigma)$ and the local strain $(\varepsilon)$ from nominal values of stress $(\mathrm{S})$ and strain (e). It is particularly more challenging when the nominal stress exceeds the local cyclic yield strength of the material (the use of static yield strength is not appropriate for steels exhibiting cyclic softening as in the case of GA 590R). Over the years, several approximate analytical methods have been advanced, none too satisfactory [16, 17]. Several years ago, one of the present authors advanced the concept of estimating the local stresses from smooth specimen strain controlled fatigue data for estimating the fatigue strength reduction factor $\left(\mathrm{K}_{\mathrm{f}}\right)$ for mechanical notches [18]. In this study, these concepts for estimating the local stresses are extended to both homogeneous and heterogeneous LWB combinations.

The basic premise of the methodology is that the fatigue life of a specimen with a laser weld joint (LWJ) under nominal stress control and that of a smooth specimen undergoing the simulated local strain reversals are the same. Then, the local cyclic stress for the smooth specimen giving the same life as that of a specimen with LWJ can be estimated from the strain controlled fatigue parameters of the base material. The equations used to estimate these are:

$$
\begin{aligned}
& \sigma_{\mathrm{n}}=\sigma_{\mathrm{f}}{ }^{\prime}\left(2 \mathrm{~N}_{\mathrm{f}}\right)^{\mathrm{b}} \\
& \sigma_{\mathrm{n}}=\mathrm{k}^{\prime}\left(\varepsilon_{\mathrm{f}}\right)^{\mathrm{n}}{ }^{\prime}\left(2 \mathrm{~N}_{\mathrm{f}}\right)^{\mathrm{cn}},
\end{aligned}
$$

The two equations are equivalent, the first equation uses the elastic strain - life parameters and the second makes use of the plastic strain -life and cyclic stress strain parameters. Obviously, the errors in estimating these parameters from experimental data (see table 4 for $\mathrm{R}^{2}$ values) will result in slightly different notch root stress values $\left(\sigma_{\mathrm{n}}\right)$. The ratio of the two stresses $\left(\sigma_{\mathrm{n}} / \mathrm{S}\right)$ yields the fatigue notch factor, $\mathrm{K}_{\mathrm{f}}$.

In analyzing the data for LWJ under nominal stress control, the run-out data were excluded for this portion of the analysis. Regression analyses were carried out on finite life data (on transformed variables) to obtain Basquin type coefficients. Figure 11 represents such data for the heterogeneous LWJ condition. The calculated $\mathrm{K}_{\mathrm{f}}$ values at several life levels are summarized in Table 6 for homogeneous and heterogeneous LWJ conditions using the elastic life parameters of the base steel to estimate the local stresses. The use of the plastic - life parameters for estimating the local stresses will give slightly different $K_{f}$ numbers, but the general trend remains the same.

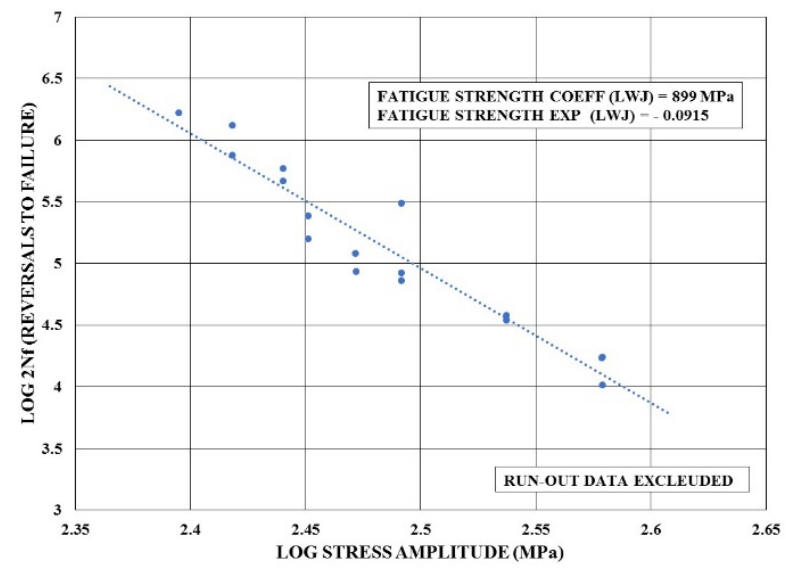

Fig.11. Linearized S-N plot for Heterogeneous LW J of GA 590R

Table 6. Fatigue notch factor for laser welded GA 590R

\begin{tabular}{|c|c|c|c|c|c|c|c|}
\hline & \multicolumn{2}{|c|}{$\begin{array}{c}\text { FATIGUE } \\
\text { PARAMETERS }\end{array}$} & \multicolumn{5}{c|}{$\begin{array}{c}\text { STRESS AMPLITUDE (MPa) FOR FATIGUE LIFE } \\
\text { (REVERSAL TO FAILURE) }\end{array}$} \\
\hline STEEL CONDITION & $\sigma_{\mathrm{f}}$ & $\mathrm{b}$ & 20,000 & 100,000 & 200,000 & $1,000,000$ & $2,000,000$ \\
\hline GA 590 MONOLITHIC & 849 & -0.0686 & 430 & 385 & 367 & 329 & 314 \\
\hline & & & & & & & \\
\hline HOMOGENEOUS LWB & 1058 & -0.0922 & 425 & 366 & 343 & 296 & 278 \\
\hline HETEROGENEOUS LWB & 899 & -0.0915 & 363 & 314 & 294 & 254 & 238 \\
\hline FATIGUE NOTCH FACTOR & & & & & & & \\
\hline $\mathrm{K}_{\mathrm{f}}^{\text {HOMO }}$ & & & 1.01 & 1.05 & 1.07 & 1.11 & 1.13 \\
\hline $\mathrm{K}_{\mathrm{f}}^{\text {HETERO }}$ & & & 1.18 & 1.23 & 1.25 & 1.30 & 1.32 \\
\hline
\end{tabular}

Figure 12 summarizes the variation of $\mathrm{K}_{\mathrm{f}}$ with fatigue life for both homogeneous and heterogeneous LWJ 
conditions. It is worth noting that $\mathrm{K}_{\mathrm{f}}$ is not a constant, but rather that it increases with increasing fatigue life and tends to "saturate" beyond about $10^{6}$ cycles to failure.

This trend is similar to that reported for several high strength sheet steels with geometric notches [18]; however, the variation of $\mathrm{K}_{\mathrm{f}}$ as a function of life appears to be both material and notch geometry $\left(\mathrm{K}_{\mathrm{t}}\right)$ dependent and as yet generalizations are difficult [13]; determination of $\mathrm{K}_{\mathrm{t}}$ to represent the laser welding process is even more difficult.

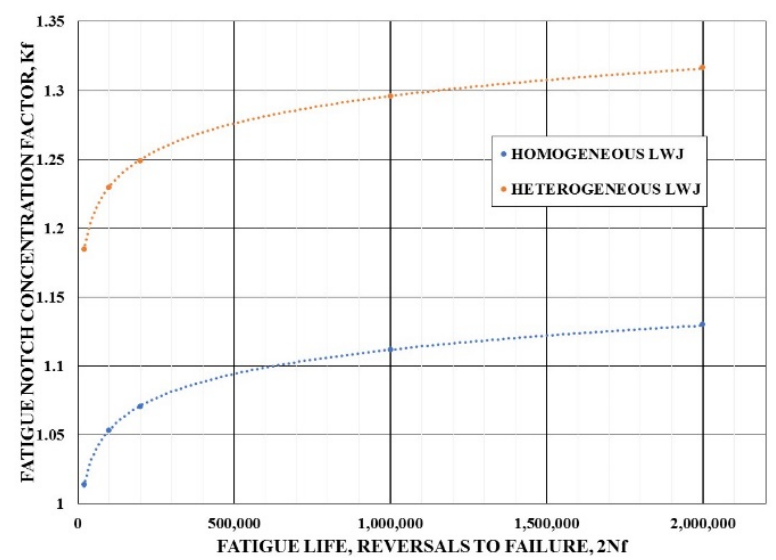

Fig.12. $\mathrm{K}_{\mathrm{f}}$ vs fatigue life for LWB of GA $590 \mathrm{R}$

While $\mathrm{K}_{\mathrm{f}}$ depends on life, it is instructive to compare the values at one life for the two LWJ combinations. For example, at $10^{5}$ cycles to failure (or $2 \times 10^{5}$ reversals), $\mathrm{K}_{\mathrm{f}}$ can be considered as 1 for the monolithic GA 590R (base steel) and it increases to 1.07 for the homogeneous $(1.4 / 1.4 \mathrm{~mm})$ and to 1.25 for the heterogeneous $(1.4 / 1.0$ $\mathrm{mm}$ ) laser welded joint configurations. In other words, while LWJ by itself reduces the fatigue strength by $7 \%$, when this technology is used to weld two gauge combinations (differential of $0.4 \mathrm{~mm}$ ), the fatigue strength decreases by $25 \%$ at the same life level. In this investigation, the same laser welding process protocols were used for both homogeneous and heterogeneous welds, and further optimization of the process (especially for heterogeneous combination) may provide different results. In addition, the present data suggest that decreasing the gauge differential, (e.g.1.4/1.2 mm combination) may also decrease the $\mathrm{K}_{\mathrm{f}}$ value for the joint, but the weight reduction potential will go down as well. Of course, another approach might be to look at different steel grades (higher strength or better ductility) to improve the base fatigue properties as well as "decrease" the LWJ notch sensitivity. Further research efforts are required to provide specific guidelines to these questions.

Working with a component supplier, strain controlled fatigue parameters and $\mathrm{K}_{\mathrm{f}}$ values for the heterogeneous geometry were utilized in a preliminary durability analysis using the commercially available CAE codes. Reasonable agreements with component testing and CAE predictions were obtained. Thus, the LWB concept for automotive chassis applications and durability assessment protocols with LWJ appear to have potential.

\section{Summary and Conclusions}

The aim of this research work was to examine the potential of LWB concepts for chassis applications, where durability concerns have been a major hurdle. The experimental program consisted of laboratory fatigue testing of the base steel and the homogeneous and heterogeneous laser welded blank combinations of two different gauges and modeling the Laser Welded Joint (LWJ) as a "notch" to determine the fatigue notch factors. The salient findings include:

1) Yield and tensile strengths of both homogeneous (same gauge) and heterogeneous LWJs are comparable to that of the base metal GA 590R (for the LWB process used here). However, the measured TE and UE are lower in both LWB conditions compared to the base metal and this apparent decrease is attributed to the hard (martensitic) weld zone in the LWJ acting as artificial "grip" region inside the gauge section of the tensile coupons.

2) For GA 590R steel, cyclic loading significantly alters the stress - strain relationship from its virgin form. The steel exhibits a large cyclic softening, for example, the cyclic yield strength of $376 \mathrm{MPa}$ is about $118 \mathrm{MPa}$ lower than the monotonic yield strength. Thus, the use of the tensile stress - strain data under fatigue loading conditions may be too optimistic.

3) Axial, fully reversed Strain - Life and Stress Life data for monolithic GA 590R (1.4 mm), and axial, fully reversed Stress - Life data for the homogeneous $(1.4 / 1.4 \mathrm{~mm})$ and heterogeneous $(1.4 / 1.0 \mathrm{~mm})$ combinations of LWJs were carried out in this investigation. The test program $(\mathrm{R}=-1)$ is unique for not introducing residual stresses during cyclic loading for characterizing the LWB concept for fatigue resistance.

4) Fatigue limit of GA 590R of $310 \mathrm{MPa}$ decreases to $300 \mathrm{MPa}$ for homogeneous and to $240 \mathrm{MPa}$ for heterogeneous LWJ configurations.

5) Central essence of this program has been to conceptualize LWJ as a "notch" which allows the estimation of local stresses at LWJ from strain - life data of the base steel and subsequently relate them to the nominal stress - life data of LWB coupons. From these, fatigue notch factors $\left(\mathrm{K}_{\mathrm{f}}\right)$ for both homogeneous and heterogeneous LWJs have been determined. Such estimation of $\mathrm{K}_{\mathrm{f}}$ is unique for fatigue work with laser welding.

6) For both homogeneous and heterogeneous LWJ configurations, $\mathrm{K}_{\mathrm{f}}$ increases with fatigue life, but tends to saturate at life levels greater than about one million cycles; thus, the use of a single constant in durability estimation regardless of life levels may lead to significant errors.

\section{Acknowledgements}

Authors acknowledge the support received from F.tech R\&D North America, during this investigation. It is also a pleasure to acknowledge the diligent work of George Girman throughout the experimental program. Permission of ArcelorMittal is highly appreciated. 


\section{References}

1. E. Bayraktar, D. Kaplan, and B. S. Yilbas, J. Mater Process Technol, 204, 440 (2008).

2. D. Anand, D. L. Chen, S. D. Bhole, P. Andreychuk and G. Boudreau, Mater Sci and Engg, A 420, 199 (2006)

3. M. R. Mitchell, Fatigue and Microstructure, (ASM, Metals Park, Ohio 1979)

4. P. C. Wang and K. M. Ewing, Welding Journal 70, (No. 10), 43 (1991)

5. S. Daneshpour, M. Kocak, S. Riekehr, and C. Gerristen, Welding in the World, 53 (no. 9/10), R221 (2009)

6. K. Asim, K. Sripichai and J. Pan, Int J Fatigue, 61, 283 (2014)

7. D. Bhattacharya, N. Fonstein, O. Girina, I. Gupta, O. Yakubovsky, $45^{\text {th }}$ MWSP Conf Proceedings (ISS, Warrendale, $\mathrm{Pa}, 2003$ )

8. A. R. Mardera, Prog Mater Sci. 45, 191 (2000)

9. M. Ono, A. Yoshitake and M. Ohmura: NLL Tech Review, 86, 13 (July 2002)

10. H. Gong, S. Wang, P. Knysh and Y. Korkolis, Mater and Design; 90, 1115 (2016)

11. D. Dong, Y. Liu, Y. Yang, J. Li, M. Ma, and T. Jian, Mater Sci and Engg. A 594, 17 (2014)

12. S. P. Bhat, HSLA Steels Technology \& Applications (ASM, Metals park, OH, 1983)

13. B. M. Wundt: Effect of Notches on Low-cycle Fatigue: A Literature Survey (ASTM, Philadelphia, PA, 1972)

14. N. E. Frost, K. J. Marsh and L. P. Pook, Metal Fatigue (Clarendon Press, 1974)

15. H. O. Fuchs and R. I. Stephens, Metal Fatigue in Engineering (John Wiley \& Sons, 1980)

16. T. H. Topper, R. M. Wetzel, and Jo Dean Morrow J Materials, 4, 200 (1969)

17. K. Molski and G. Glinka, Mater Sci Engg, 50, 93 (1981)

18. S. P. Bhat, High Strength Steel for Automotive Use (SAE, Warrendale, PA 1983) 